PROCEEDINGS OF THE

AMERICAN MATHEMATICAL SOCIETY

Volume 137, Number 1, January 2009, Pages 161-169

S 0002-9939(08)09612-3

Article electronically published on July 1, 2008

\title{
RADIAL SOLUTIONS FOR SOME NONLINEAR PROBLEMS INVOLVING MEAN CURVATURE OPERATORS IN EUCLIDEAN AND MINKOWSKI SPACES
}

\author{
C. BEREANU, P. JEBELEAN, AND J. MAWHIN \\ (Communicated by Carmen C. Chicone)
}

\begin{abstract}
In this paper, using the Schauder fixed point theorem, we prove existence results of radial solutions for Dirichlet problems in the unit ball and in an annular domain, associated to mean curvature operators in Euclidean and Minkowski spaces.
\end{abstract}

\section{INTRODUCTION}

The aim of this paper is to present some existence results of radial solutions for Dirichlet problems in the unit ball and in an annular domain, associated to mean curvature operators in Euclidean and Minkowski spaces. Those problems are classical in the Euclidean space (see e.g. 8]). In the flat Minkowski space $\mathbb{L}^{N+1}=\left\{(x, t): x \in \mathbb{R}^{N}, t \in \mathbb{R}\right\}$, those problems originate from studying, in differential geometry or relativity, maximal or constant mean curvature hypersurfaces, i.e. spacelike submanifolds of codimension one in $\mathbb{L}^{N+1}$, having the property that their mean extrinsic curvature (trace of its second fundamental form) is respectively zero or constant.

More specifically, when the searched $N$-dimensional hypersurface in $\mathbb{L}^{N+1}$ can be represented as the graph of a function $v: \Omega \rightarrow \mathbb{R}$ with $\Omega$ a bounded domain in $\left\{(x, t) \in \mathbb{L}^{N+1}: t=0\right\} \simeq \mathbb{R}^{N}$, one is led, given $H: \Omega \times \mathbb{R} \rightarrow \mathbb{R}$ (which is zero for maximal hypersurfaces and constant for hypersurfaces of constant mean curvature), to maximize an integral of the form

$$
I(v)=\int_{\Omega}\left[\sqrt{1-|\nabla v(x)|^{2}}-\int_{0}^{v(x)} H(x, t) d t\right] d x
$$

over a suitable class of functions $v$ depending upon the boundary conditions. The corresponding Euler-Lagrange equation has the form

$$
\operatorname{div}\left(\frac{\nabla v(x)}{\sqrt{1-|\nabla v(x)|^{2}}}\right)=H(x, v(x))
$$

Received by the editors December 4, 2007.

2000 Mathematics Subject Classification. Primary 35J65; Secondary 34B15.

Key words and phrases. Mean curvature operator, Minkowski space, radial solution, Schauder fixed point theorem.

(C)2008 American Mathematical Society 
and we refer to [1] for a discussion, earlier references and existence results when $\Omega$ is arbitrary and $H$ is bounded.

More precisely, the problems we consider here are of the type

$$
\operatorname{div}\left(\phi_{N}(\nabla v)\right)=f\left(|x|, v, \frac{d v}{d r}\right) \quad \text { in } \quad \Omega, \quad v=0 \quad \text { on } \quad \partial \Omega
$$

where

$$
\phi_{N}(y)=\frac{y}{\sqrt{1 \pm|y|^{2}}} \quad\left(y \in \mathbb{R}^{N}\right)
$$

with the + sign in the Euclidean case, the - sign in the Minkowski case, $\Omega$ denotes the unit ball $\mathcal{B} \subset \mathbb{R}^{N}$ or an annular domain $\mathcal{A}=\left\{x \in \mathbb{R}^{N}: 1<|x|<2\right\}$, the function $f$ is continuous, $|\cdot|$ denotes the Euclidean norm in $\mathbb{R}^{N}$ and $\frac{d v}{d r}$ stands for the radial derivative of $v$. Setting $|x|=r$ and $v(x)=u(r)$, the above Dirichlet problem becomes

$$
\left(r^{N-1} \phi_{1}\left(u^{\prime}\right)\right)^{\prime}=r^{N-1} f\left(r, u, u^{\prime}\right), \quad b\left(u, u^{\prime}\right)=0,
$$

where $b\left(u, u^{\prime}\right)=0$ denotes the mixed boundary condition $u^{\prime}(0)=0=u(1)$ or the Dirichlet boundary condition $u(1)=0=u(2)$, according to whether $\Omega$ equals $\mathcal{B}$, respectively $\mathcal{A}$. Notice that (1.1) need not be the Euler-Lagrange equations of a variational problem.

For this reason, (1.2) is transformed into a fixed point problem to which we apply the Schauder fixed point theorem. Notice that in the Euclidean space situation, the corresponding fixed point operator is not defined on the whole space and we overcome this difficulty by using a cutting method introduced in [2]. For other results concerning the Euclidean situation, see for example 4]. In the Minkowski setting, we prove that the problem is solvable for an arbitrary continuous right-hand member $f$. The case where $N=1$ was already considered in $[3]$.

\section{Radial SOLUtions IN THE UNit BALL}

In this section, $\mathcal{B}$ denotes the open unit ball in $\mathbb{R}^{N}$ and $f:[0,1] \times \mathbb{R}^{2} \rightarrow \mathbb{R}$ is a continuous function. The first main result concerns the existence of classical radial solutions of the nonlinear Dirichlet problem associated with the mean extrinsic curvature operator in Minkowski space

$$
\operatorname{div}\left(\frac{\nabla v}{\sqrt{1-|\nabla v|^{2}}}\right)=f\left(|x|, v, \frac{d v}{d r}\right) \quad \text { in } \quad \mathcal{B}, \quad v=0 \quad \text { on } \quad \partial \mathcal{B} .
$$

We have for (2.1) the following 'universal' existence result.

Theorem 2.1. Problem (2.1) has at least one classical radial solution for any continuous right-hand member $f$.

Notice that, when $f(r, u, v)=H$ (case of constant mean extrinsic curvature), the radial solution of (2.1) is unique and explicitly given, for any $H \in \mathbb{R}$, by

$$
u(r)=0 \quad(H=0), \quad u(r)=\frac{N}{H}\left[\sqrt{1+\frac{H^{2}}{N^{2}} r^{2}}-\sqrt{1+\frac{H^{2}}{N^{2}}}\right] \quad(H \neq 0) .
$$

The second main result of the section deals with the existence of classical radial solutions of the nonlinear Dirichlet problem associated with the mean curvature 
operator in Euclidean space

$$
\operatorname{div}\left(\frac{\nabla v}{\sqrt{1+|\nabla v|^{2}}}\right)=f\left(|x|, v, \frac{d v}{d r}\right) \quad \text { in } \quad \mathcal{B}, \quad v=0 \quad \text { on } \quad \partial \mathcal{B} .
$$

Theorem 2.2. Assume that there exists $\alpha>0$ such that $\alpha / N<1$ and

$$
|f(r, u, v)| \leq \alpha \quad \text { for all } \quad(r, u, v) \in[0,1] \times R_{\alpha},
$$

where $R_{\alpha}$ is the square given by $R_{\alpha}=\left[-\frac{\alpha / N}{\sqrt{1-(\alpha / N)^{2}}}, \frac{\alpha / N}{\sqrt{1-(\alpha / N)^{2}}}\right]^{2}$. Then, problem (2.2) has at least one classical radial solution.

Notice that, when $f(r, u, v)=H$ (case of constant mean curvature), the radial solution of (2.2) only exists if $|H|<N$, is unique and is explicitly given by

$$
u(r)=0 \quad(H=0), \quad u(r)=\frac{N}{H}\left[\sqrt{1-\frac{H^{2}}{N^{2}}}-\sqrt{1-\frac{H^{2}}{N^{2}} r^{2}}\right] \quad(0<|H|<N) .
$$

Hence condition $\alpha / N<1$ in Theorem 2.2 is sharp.

When dealing with the radial solutions for (2.1) or (2.2), one is led to study (setting $|x|=r$ and $v(x)=u(r)$ ) the mixed boundary-value problem

$$
\left(r^{N-1} \phi\left(u^{\prime}\right)\right)^{\prime}=r^{N-1} f\left(r, u, u^{\prime}\right), \quad u^{\prime}(0)=0=u(1),
$$

where $\phi(y)=\frac{y}{\sqrt{1-y^{2}}}$ in the Minkowski case and $\phi(y)=\frac{y}{\sqrt{1+y^{2}}}$ in the Euclidean case $(y \in \mathbb{R})$.

We first reformulate (2.3) as a fixed point problem, for a general class of $\phi$ containing the first example above as special cases, namely $\phi:(-a, a) \rightarrow \mathbb{R}$ an increasing homeomorphism such that $\phi(0)=0$ and $0<a \leq \infty$. In this section $C$ stands for the Banach space of continuous functions defined on $[0,1]$ endowed with the usual sup-norm $\|\cdot\|_{\infty}$ and $C^{1}$ denotes the Banach space of continuously differentiable functions on $[0,1]$ equipped with the norm $\|u\|=\|u\|_{\infty}+\left\|u^{\prime}\right\|_{\infty}$. The subspaces of $C^{1}$ defined by

$$
C_{M}^{1}=\left\{u \in C^{1}: u^{\prime}(0)=0=u(1)\right\}
$$

and

$$
C_{0}=\{u \in C: u(0)=0\}
$$

are closed. Then, setting

$$
\gamma(r)=1 / r^{N-1} \quad(r>0)
$$

consider the linear operators

$$
\begin{gathered}
S: C \rightarrow C_{0}, \quad S u(r)=\gamma(r) \int_{0}^{r} t^{N-1} u(t) d t \quad(r \in(0,1]), \\
K: C \rightarrow C^{1}, \quad K u(r)=\int_{1}^{r} u(t) d t \quad(r \in[0,1]) .
\end{gathered}
$$

It is easy to see that $K$ is a bounded operator and standard arguments, invoking the Arzela-Ascoli theorem, show that $S$ is compact. Now, let $N_{f}: C^{1} \rightarrow C$ be the Nemytskii operator associated to $f$, defined by

$$
N_{f}(u)=f\left(\cdot, u(\cdot), u^{\prime}(\cdot)\right) \quad \forall u \in C^{1} .
$$


Note that $N_{f}$ is continuous and takes bounded sets into bounded sets. The following result has been proved in [7] if $a=\infty$. The proof for the case $a<\infty$ is completely similar to the one given in [7] and, actually, can be easily deduced from the properties of the above operators.

Lemma 2.3. The nonlinear operator

$$
\mathcal{M}: C_{M}^{1} \rightarrow C_{M}^{1}, \quad \mathcal{M}=K \circ \phi^{-1} \circ S \circ N_{f}
$$

is well defined, compact, and $u \in C_{M}^{1}$ is a solution of (2.3) if and only if $\mathcal{M}(u)=u$.

Proposition 2.4. Assume that $0<a<\infty$ and $\phi:(-a, a) \rightarrow \mathbb{R}$ is an increasing homeomorphism such that $\phi(0)=0$. Then, problem (2.3) has at least one solution.

Proof. Let $u \in C_{M}^{1}$ and $v=\mathcal{M}(u)$. It follows that

$$
\left\|v^{\prime}\right\|_{\infty}=\left\|\phi^{-1} \circ S \circ N_{f}(u)\right\|_{\infty}<a .
$$

From (2.4) and

$$
\|v\|_{\infty}=\left\|K\left(v^{\prime}\right)\right\|_{\infty}
$$

it follows that

$$
\|v\|_{\infty}<a .
$$

Hence,

$$
\|v\|<2 a \text {. }
$$

From the above estimate and the Schauder fixed point theorem, we deduce that there exist $u \in C_{M}^{1}$ such that $u=\mathcal{M}(u)$. Using Lemma 2.3. it follows that $u$ is also a solution of (2.3).

Proposition 2.5. Let $0<a \leq \infty$ and $\phi: \mathbb{R} \rightarrow(-a, a)$ be an increasing homeomorphism such that $\phi(0)=0$. If there exists $\alpha>0$ such that $\alpha / N<a$ and

$$
|f(r, u, v)| \leq \alpha \quad \text { for all } \quad(r, u, v) \in[0,1] \times R_{\alpha}(\phi),
$$

where $R_{\alpha}(\phi)$ is the rectangle given by

$$
\left.R_{\alpha}(\phi)=\left[-\phi^{-1}(\alpha / N)\right),-\phi^{-1}(-\alpha / N)\right] \times\left[\phi^{-1}(-\alpha / N), \phi^{-1}(\alpha / N)\right],
$$

then problem (2.3) has at least one solution $u \in \Omega_{\alpha}(\phi)$, where

$$
\Omega_{\alpha}(\phi)=\left\{u \in C_{M}^{1}:\left(u(r), u^{\prime}(r)\right) \in R_{\alpha}(\phi), \quad \forall r \in[0,1]\right\} .
$$

Proof. We distinguish two cases.

The case $a=\infty$. We show that

$$
\mathcal{M}\left(\Omega_{\alpha}(\phi)\right) \subset \Omega_{\alpha}(\phi),
$$

where $\mathcal{M}$ is the fixed point operator associated to (2.3) (see Lemma 2.3). Let $u \in \Omega_{\alpha}(\phi)$ and $v=\mathcal{M}(u)$. Using (2.5), it follows that

$$
\left|\phi\left(v^{\prime}(r)\right)\right|=\left|\gamma(r) \int_{0}^{r} t^{N-1} f\left(t, u(t), u^{\prime}(t)\right) d t\right| \leq \alpha / N
$$

for all $r \in(0,1]$, and because $\phi\left(v^{\prime}(0)\right)=0$, the homeomorphic character of $\phi$ implies that

$$
v^{\prime}(r) \in\left[\phi^{-1}(-\alpha / N), \phi^{-1}(\alpha / N)\right] \text { for all } r \in[0,1] .
$$


Hence, using $v=K\left(v^{\prime}\right)$, we deduce that

$$
\left.v(r) \in\left[-\phi^{-1}(\alpha / N)\right),-\phi^{-1}(-\alpha / N)\right] \text { for all } r \in[0,1] .
$$

Consequently, $v \in \Omega_{\alpha}(\phi)$ and (2.6) is proved. Now, using the fact that $\Omega_{\alpha}(\phi)$ is a closed convex set in $C_{M}^{1}$ invariant for the compact operator $\mathcal{M}$, it follows by the Schauder fixed point theorem that there exists $u \in \Omega_{\alpha}(\phi)$ such that $\mathcal{M}(u)=u$, which is also a solution of (2.3).

The case $a<\infty$. Since $\alpha / N<a$, we can construct an increasing homeomorphism $\psi: \mathbb{R} \rightarrow \mathbb{R}$ such that

$$
\psi(u)=\phi(u) \quad \text { for all } \quad u \in\left[\phi^{-1}(-\alpha / N), \phi^{-1}(\alpha / N)\right] .
$$

It is clear that $R_{\alpha}(\phi)=R_{\alpha}(\psi)$ and $\Omega_{\alpha}(\phi)=\Omega_{\alpha}(\psi)$. Hence, by the first step, problem

$$
\left(r^{N-1} \psi\left(u^{\prime}\right)\right)^{\prime}=r^{N-1} f\left(r, u, u^{\prime}\right), \quad u^{\prime}(0)=0=u(1),
$$

has at least one solution $u \in \Omega_{\alpha}(\psi)$, which is also a solution of (2.3).

The proofs of Theorems 2.1 and 2.2. Taking $v(x)=u(|x|)$ for all $x \in \mathcal{B}$, we have that Theorem 2.1 follows from Proposition 2.4 (with $\phi(u)=\frac{u}{\sqrt{1-u^{2}}}$ ) and Theorem 2.2 follows from Proposition $2.5\left(\right.$ with $\left.\phi(u)=\frac{u}{\sqrt{1+u^{2}}}\right)$.

Remark 2.6. Taking in Proposition 2.5, $\phi(u)=|u|^{p-2} u(p>1)$ and $v(x)=u(|x|)$ for all $x \in \overline{\mathcal{B}}$, we recover an existence result already proved in $[5]$.

When $f$ is independent of $\frac{d u}{d r}$, it is easy to formulate simple uniqueness conditions for the solution of (2.3), and hence for the radial solutions of (2.1) and (2.2).

Theorem 2.7. If $f:[0,1] \times \mathbb{R} \rightarrow \mathbb{R}$ is such that $f(r, \cdot)$ is nondecreasing for each fixed $r \in[0,1]$, then problem

$$
\left(r^{N-1} \phi\left(u^{\prime}\right)\right)^{\prime}=r^{N-1} f(r, u), \quad u^{\prime}(0)=0=u(1)
$$

has at most one solution, and the same is true for the radial solutions of problems

$$
\operatorname{div}\left(\frac{\nabla v}{\sqrt{1-|\nabla v|^{2}}}\right)=f(|x|, v) \quad \text { in } \quad \mathcal{B}, \quad v=0 \quad \text { on } \quad \partial \mathcal{B}
$$

and

$$
\operatorname{div}\left(\frac{\nabla v}{\sqrt{1+|\nabla v|^{2}}}\right)=f(|x|, v) \quad \text { in } \quad \mathcal{B}, \quad v=0 \quad \text { on } \quad \partial \mathcal{B} .
$$

Proof. Assume that $u$ and $w$ are solutions of (2.7), and that $u \neq w$. It follows from the boundary conditions that $E:=\left\{r \in[0,1]: u^{\prime}(r) \neq w^{\prime}(r)\right\}$ has positive measure. Now, multiplying the identity

$$
\left[r^{N-1}\left(\phi\left(u^{\prime}\right)-\phi\left(w^{\prime}\right)\right)\right]^{\prime}=r^{N-1}[f(r, u)-f(r, w)]
$$

by $u-w$, integrating over $[0,1]$, integrating by parts and using the boundary conditions and the increasing character of $\phi$, we get

$$
\begin{aligned}
0 & >-\int_{E}\left[\phi\left(u^{\prime}(r)\right)-\phi\left(w^{\prime}(r)\right)\right]\left[u^{\prime}(r)-w^{\prime}(r)\right] r^{N-1} d r \\
& =\int_{0}^{1}[f(r, u(r))-f(r, w(r))][u(r)-w(r)] d r \geq 0,
\end{aligned}
$$

a contradiction. 


\section{RADIAL SOLUTIONS IN AN ANNULAR DOMAIN}

In this section, $\mathcal{A}$ denotes the annular domain $\left\{x \in R^{N}: 1<|x|<2\right\}$ and $f:[1,2] \times \mathbb{R}^{2} \rightarrow \mathbb{R}$ is a continuous function. The first main result concerns the existence of classical radial solutions of the nonlinear Dirichlet problem associated with the mean extrinsic curvature operator in Minkowski space

$$
\operatorname{div}\left(\frac{\nabla v}{\sqrt{1-|\nabla v|^{2}}}\right)=f\left(|x|, v, \frac{d v}{d r}\right) \quad \text { in } \quad \mathcal{A}, \quad v=0 \quad \text { on } \quad \partial \mathcal{A} .
$$

We have the following 'universal' existence result.

Theorem 3.1. Problem (3.1) has at least one classical radial solution for any continuous right-hand member $f$.

The following result concerns the existence of radial solutions for the nonlinear Dirichlet problem associated with the mean curvature operator in Euclidean space

$$
\operatorname{div}\left(\frac{\nabla v}{\sqrt{1+|\nabla v|^{2}}}\right)=f\left(|x|, v, \frac{d v}{d r}\right) \quad \text { in } \quad \mathcal{A}, \quad v=0 \quad \text { on } \partial \mathcal{A} \text {. }
$$

Theorem 3.2. Assume that there exists $\alpha>0$ such that $\alpha_{N}:=\frac{2 \alpha\left(2^{N}-1\right)}{N}<1$ and

$$
|f(r, u, v)| \leq \alpha \quad \text { for all } \quad(r, u, v) \in[1,2] \times P_{\alpha},
$$

where $P_{\alpha}$ is the square given by $P_{\alpha}=\left[-\frac{\alpha_{N}}{\sqrt{1-\alpha_{N}^{2}}}, \frac{\alpha_{N}}{\sqrt{1-\alpha_{N}^{2}}}\right]^{2}$. Then, problem (3.2) has at least one classical radial solution.

When dealing with the radial solutions for problems (3.1) or (3.2), we are led to consider the nonlinear Dirichlet problem

$$
\left(r^{N-1} \phi\left(u^{\prime}\right)\right)^{\prime}=r^{N-1} f\left(r, u, u^{\prime}\right), \quad u(1)=0=u(2),
$$

where $\phi(u)=\frac{u}{\sqrt{1-u^{2}}}$ or $\phi(u)=\frac{u}{\sqrt{1+u^{2}}}$, respectively.

As in the preceding section we start with the case where $\phi:(-a, a) \rightarrow \mathbb{R}$ is an increasing homeomorphism such that $\phi(0)=0$ and $0<a \leq \infty$. In this situation we reformulate (3.3) as a fixed point problem.

In this section $C$ stands for the Banach space of continuous functions defined on $[1,2]$ endowed with the norm $\|\cdot\|_{\infty}$. On the other hand, $C^{1}$ denotes the Banach space of continuously differentiable functions on $[1,2]$ equipped with the norm $\|u\|=$ $\|u\|_{\infty}+\left\|u^{\prime}\right\|_{\infty}$ and $C_{D}^{1}$ denotes the closed subspace of $C^{1}$ defined by

$$
C_{D}^{1}=\left\{u \in C^{1}: u(1)=0=u(2)\right\} .
$$

Consider the linear operators

$$
\begin{gathered}
L: C \rightarrow C, \quad L u(r)=\gamma(r) \int_{1}^{r} t^{N-1} u(t) d t \quad(r \in[1,2]), \\
H: C \rightarrow C^{1}, \quad H u(r)=\int_{1}^{r} u(t) d t \quad(r \in[1,2]) .
\end{gathered}
$$

It is not difficult to prove that $H$ is a bounded operator and $L$ is compact. Then, let $N_{f}$ be the Nemitskii operator associated to $f$ defined as in the previous section. The following lemma is the key ingredient used in the construction of the fixed point operator associated to (3.3). 
Lemma 3.3. For each $h \in C$ there exists a unique $\alpha:=Q_{\phi}(h) \in \mathbb{R}$ such that

$$
\int_{1}^{2} \phi^{-1}\left(h(r)-Q_{\phi}(h) \gamma(r)\right) d r=0
$$

Moreover, the function $Q_{\phi}: C \rightarrow \mathbb{R}$ is continuous and satisfies

$$
\left|Q_{\phi}(h)\right| \leq\|h / \gamma\|_{\infty} \text { for all } h \in C .
$$

Proof. Let $h \in C$. We first prove uniqueness. Let $\alpha_{i} \in \mathbb{R}$ be such that

$$
\int_{1}^{2} \phi^{-1}\left(h(r)-\alpha_{i} \gamma(r)\right) d r=0 \quad(i=1,2) .
$$

It follows that there exists $r_{0} \in[1,2]$ such that

$$
\phi^{-1}\left(h\left(r_{0}\right)-\alpha_{1} \gamma\left(r_{0}\right)\right)=\phi^{-1}\left(h\left(r_{0}\right)-\alpha_{2} \gamma\left(r_{0}\right)\right),
$$

and using the injectivity of $\phi^{-1}$ we deduce that $\alpha_{1}=\alpha_{2}$. For the existence, it is clear that the function

$$
F:\left[-\|h / \gamma\|_{\infty},\|h / \gamma\|_{\infty}\right] \rightarrow \mathbb{R}, \quad t \mapsto \int_{1}^{2} \phi^{-1}(h(r)-t \gamma(r)) d r
$$

is continuous and $F\left(-\|h / \gamma\|_{\infty}\right) F\left(\|h / \gamma\|_{\infty}\right) \leq 0$. Hence, there exists a unique $\alpha:=$ $Q_{\phi}(h) \in\left[-\|h / \gamma\|_{\infty},\|h / \gamma\|_{\infty}\right]$ such that $F(\alpha)=0$, which means that (3.4) and (3.5) hold. The continuity of $Q_{\phi}$ follows immediately from the dominated convergence theorem.

The following result is a fixed point reformulation of (3.3) when $\phi:(-a, a) \rightarrow \mathbb{R}$ is an increasing homeomorphism such that $\phi(0)=0$ and $0<a \leq \infty$. In the case $a=\infty$, a different fixed point operator associated to (3.3) has been used in [6] in order to obtain a multiplicity result.

Lemma 3.4. Consider the nonlinear operator

$$
\mathcal{D}: C_{D}^{1} \rightarrow C_{D}^{1}, \quad \mathcal{D}=H \circ \phi^{-1} \circ\left(I-\gamma Q_{\phi}\right) \circ L \circ N_{f} .
$$

Then, $\mathcal{D}$ is well defined, compact and $u \in C_{D}^{1}$ is a solution of (3.3) if and only if $\mathcal{D}(u)=u$.

Proof. Let $u \in C_{D}^{1}$. It is clear that $\mathcal{D}(u)(1)=0$. On the other hand, applying Lemma 3.3 with $h=\left(L \circ N_{f}\right)(u)$, it follows that $\mathcal{D}(u)(2)=0$. Hence, $\mathcal{D}$ is well defined. Now, we know that the operators which compose $\mathcal{D}$ are continuous and take bounded sets into bounded sets. Moreover, the linear operator $L$ is compact. This implies the compactness of $\mathcal{D}$.

Let $u \in C_{D}^{1}$ be such that $\mathcal{D}(u)=u$. This implies that $u$ satisfies the Dirichlet boundary condition on $[1,2],\left\|u^{\prime}\right\|_{\infty}<a$ and

$$
\left.\phi\left(u^{\prime}\right)(r)=\gamma(r) \int_{1}^{r} t^{N-1} N_{f}(u)(t) d t-\gamma(r) Q_{\phi}\left[\left(L \circ N_{f}\right)(u)\right)\right]
$$

for all $r \in[1,2]$. This implies that $u$ satisfies the differential equation in (3.3). The remaining part of the proof is obvious.

The following result is an immediate consequence of the above fixed point reduction and the Schauder fixed point theorem.

Proposition 3.5. Assume that $0<a<\infty$ and $\phi:(-a, a) \rightarrow \mathbb{R}$ is an increasing homeomorphism such that $\phi(0)=0$. Then, problem (3.3) has at least one solution. 
Proof. See the proof of Proposition 2.4

Proposition 3.6. Let $\phi: \mathbb{R} \rightarrow(-a, a)$ be an increasing homeomorphism such that $\phi(0)=0$ and $0<a \leq \infty$. If there exists $\alpha>0$ such that $\alpha_{N}:=\frac{2 \alpha\left(2^{N}-1\right)}{N}<a$ and

$$
|f(r, u, v)| \leq \alpha \quad \text { for all } \quad(r, u, v) \in[1,2] \times R_{\alpha}(\phi),
$$

where $R_{\alpha}(\phi)$ is the square given by

$$
R_{\alpha}(\phi)=\left[\phi^{-1}\left(-\alpha_{N}\right), \phi^{-1}\left(\alpha_{N}\right)\right]^{2},
$$

then problem (3.3) has at least one solution $u \in \Omega_{\alpha}(\phi)$, where

$$
\Omega_{\alpha}(\phi)=\left\{u \in C_{D}^{1}:\left(u(r), u^{\prime}(r)\right) \in R_{\alpha}(\phi), \quad \forall r \in[1,2]\right\} .
$$

Proof. We distinguish two cases.

The case $a=\infty$. We show that

$$
\mathcal{D}\left(\Omega_{\alpha}(\phi)\right) \subset \Omega_{\alpha}(\phi),
$$

where $\mathcal{D}$ is the fixed point operator associated to (3.3) (see Lemma 3.4). Let $u \in \Omega_{\alpha}(\phi)$ and $v=\mathcal{D}(u)$. It follows that

$$
\phi\left(v^{\prime}\right)=\left(L \circ N_{f}\right)(u)-\gamma Q_{\phi}\left[\left(L \circ N_{f}\right)(u)\right] .
$$

Using Lemma 3.3 and (3.6), we infer

$$
\left\|\gamma Q_{\phi}\left[\left(L \circ N_{f}\right)(u)\right]\right\|_{\infty} \leq \alpha_{N} / 2 .
$$

Hence

$$
\left\|\phi\left(v^{\prime}\right)\right\|_{\infty} \leq \alpha_{N}
$$

This implies that $v \in \Omega_{\alpha}(\phi)$ and (3.7) holds. The result follows now from the Schauder fixed point theorem.

The case $a<\infty$. To prove the result in this case, use the first step and similar arguments as in the proof of Proposition 2.5.

The proofs of Theorems 3.1 and 3.2. Taking $v(x)=u(|x|)$ for all $x \in \overline{\mathcal{A}}$, we have that Theorem 3.1 follows from Proposition $3.5\left(\right.$ with $\left.\phi(u)=\frac{u}{\sqrt{1-u^{2}}}\right)$ and Theorem 3.2 follows from Proposition 3.6 (with $\phi(u)=\frac{u}{\sqrt{1+u^{2}}}$ ).

When $f$ is independent of $\frac{d u}{d r}$, proceeding exactly as in Section 2, we can prove the following simple uniqueness conditions for the solution of (3.3), and hence for the radial solution of (3.1) and of (3.2).

Theorem 3.7. If $f:[0,1] \times \mathbb{R} \rightarrow \mathbb{R}$ is such that $f(r, \cdot)$ is nondecreasing for each fixed $r \in[0,1]$, then problem

$$
\left(r^{N-1} \phi\left(u^{\prime}\right)\right)^{\prime}=r^{N-1} f(r, u), \quad u(1)=0=u(2)
$$

has at most one solution, and the same is true for the radial solutions in $\mathcal{A}$ of problems

$$
\operatorname{div}\left(\frac{\nabla v}{\sqrt{1-|\nabla v|^{2}}}\right)=f(|x|, v) \quad \text { in } \quad \mathcal{A}, \quad v=0 \quad \text { on } \quad \partial \mathcal{A}
$$

and

$$
\operatorname{div}\left(\frac{\nabla v}{\sqrt{1+|\nabla v|^{2}}}\right)=f(|x|, v) \quad \text { in } \quad \mathcal{A}, \quad v=0 \quad \text { on } \quad \partial \mathcal{A}
$$


Remark 3.8. As noticed by the referee, some results about (1.2) can be extended to equations of the form

$$
\left(p(r) \phi\left(u^{\prime}\right)\right)^{\prime}=p(r) f\left(r, u, u^{\prime}\right)
$$

under suitable conditions upon the positive function $p(r)$.

\section{REFERENCES}

1. R. Bartnik and L. Simon, Spacelike hypersurfaces with prescribed boundary values and mean curvature, Commun. Math. Phys. 87 (1982/83), 131-152. MR0680653 (84j:58126)

2. C. Bereanu and J. Mawhin, Periodic solutions of nonlinear perturbations of $\phi$-Laplacians with possibly bounded $\phi$, Nonlinear Analysis 68 (2008), 1668-1681. MR2388840

3. C. Bereanu and J. Mawhin, Existence and multiplicity results for some nonlinear problems with singular $\phi$-Laplacian, J. Differential Equations 243 (2007), 536-557. MR2371799

4. A. Capietto, W. Dambrosio, and F. Zanolin, Infinitely many radial solutions to a boundary value problem in a ball, Ann. Mat. Pura Appl. 179 (2001), 159-188. MR.1848759 (2002f:35097)

5. G. Dincă and P. Jebelean, Radial solutions for a nonlinear problem with p-Laplacian, Differential Integral Equations 9 (1996), 1139-1146. MR.1392098 (97c:35050)

6. M. García-Huidobro, R. Manásevich, and F. Zanolin, Strongly nonlinear second-order ODEs with rapidly growing terms, J. Math. Anal. Appl. 202 (1996), 1-26. MR1402585 (98b:34022)

7. M. García-Huidobro, R. Manásevich, and F. Zanolin, Infinitely many solutions for a Dirichlet problem with a nonhomogeneous p-Laplacian-like operator in a ball, Adv. Differential Equations 2 (1997), 203-230. MR 1424768 (97k:35074)

8. D. Gilbarg and N.S. Trudinger, Elliptic Partial Differential Equations of Second Order, Second Edition, Springer, Berlin, 1983. MR0737190 (86c:35035)

Département de Mathématique, Université Catholique de Louvain, Chemin Du Cyclotron 2, B-1348 Louvain-la-Neuve, Belgium

E-mail address: cristian.bereanu@uclouvain.be

Department of Mathematics, West University of Timişoara, Blvd. V. PÂrvan No. 4, RO-1900 Timişoara, Romania

E-mail address: jebelean@math.uvt.ro

Département de Mathématique, Université Catholique de Louvain, Chemin du Cyclotron 2, B-1348 Louvain-la-Neuve, Belgium

E-mail address: jean.mawhin@uclouvain.be 